\title{
Time to Sputum Smear Conversion and Its Predictors Among Smear-Positive Pulmonary TB Patients in East Gojjam Zone, Northwest Ethiopia: A Longitudinal Study.
}

Mulusew Andualem Asemahagn ( $\square$ muler.hi@gmail.com )

Bahir Dar University

\section{Research Article}

Keywords: Pulmonary TB, smear conversion, factors, East Gojjam, Ethiopia

Posted Date: February 17th, 2021

DOI: https://doi.org/10.21203/rs.3.rs-200228/v1

License: (9) This work is licensed under a Creative Commons Attribution 4.0 International License. Read Full License

Version of Record: A version of this preprint was published at BMC Pulmonary Medicine on April 8th, 2021. See the published version at https://doi.org/10.1186/s12890-021-01483-w. 


\section{Time to sputum smear conversion and its predictors among smear- positive pulmonary TB patients in East Gojjam Zone, Northwest Ethiopia: A longitudinal study.}

Mulusew Andualem Asemahagn $\boldsymbol{\delta}$

$\mathrm{PhD}$ in public health, associate professor, school of Public Health, College of Medicine and Health Sciences, Bahir Dar University, Bahir Dar, Ethiopia

${ }^{\boldsymbol{\delta}}$ Corresponding author

email: muler.hi@gmail.com

Address: https://www.bdu.edu.et/cmhs/content/school-public-health-staff 


\section{Abstract}

Background: Sputum smear conversion is a key indicator of treatment response and reduced infectivity among smear-positive pulmonary tuberculosis (SPPTB). This study aimed at estimating sputum smear conversion time and identifying factors hindering sputum smear conversion among SPPTB patients in East Gojjam Zone, Northwest Ethiopia.

Methods: A total of 282 SPPTB patients were followed for 22 weeks through weekly sputum smear evaluation. Due to the absence of sputum culture and rapid diagnostic services, sputum smear conversion evaluation was conducted microscopically using acid-fast-bacilli staining technique of sediments from a 5\% sodium hypochlorite concentration technique. Data on socio-demographic, clinical profile, and personal behavior variables were collected using a pretested intervieweradministered questionnaire. Various descriptive statistics including mean, median with interquartile range (IQR), proportions, and cross-tabulations were computed. Factors affecting sputum smear conversion were identified by multivariable logistic regression analysis. The statistical significance of variables was determined at a p-value $<0.05$.

Results: Over half, 166(59\%) of SPPTB cases were males and 147(52\%) were rural dwellers. The mean age of respondents was $35 \pm 5 \mathrm{SD}$ years. About $88(31.2 \%)$ of SPPTB patients had comorbidities, 102(36.2\%) faced stigma, and 54(19\%) smoked a cigarette. The median sputum smear conversion time at the end of the intensive phase was 35 days (IQR: 21 -56 days). The majority, 85\% (95\%CI: 76\% -93\%) and 95\% (95\% CI: 85\% -99\%) of SPPTB patients underwent sputum smear conversion at the end of $2^{\text {nd }}$ and $5^{\text {th }}$ months of treatment, respectively. Poor knowledge on TB, being HIV positive, higher smear grading, having diabetes mellitus, undernutrition, cigarette smoking, facing societal stigma, and TB service delay were positively associated with the length of sputum smear conversion. Conclusion: Although the treatment success rate was 95\%, the median sputum smear conversion time was higher compared to the TB program expectations and some former studies. Factors of sputum smear conversion were related to nutritional status, smear grading, comorbidity status, knowledge on $\mathrm{TB}$, personal behavior, stigma, and TB service delay. Improving the health literacy of the community by revising the existing community awareness strategies is essential to enhance treatment adherence and lower infectiousness after treatment initiation.

Keywords: Pulmonary TB, smear conversion, factors, East Gojjam, Ethiopia 


\section{Background}

Several millions of lives have been saved because of various global and national anti-TB interventions [1]. Tuberculosis, largely caused by mycobacterium tuberculosis, however, remains a global health problem with varying magnitudes across countries and regions [1,2]. Countries with poor socio-economic conditions have been affected more by TB in terms of morbidity, mortality and cost for care $[2,3]$. As a result, over 10 million new TB cases were reported in 2019 where $88 \%$ of the new TB cases were among people aged $\geq 15$ years. Over half, $56 \%$ was among adult males and there were about 1.4 million TB deaths. About $44 \%$ of the global new TB cases were from SouthEast-Asian countries followed by $25 \%$ from African countries. South Africa and Nigeria are among the top eight high TB burden countries [1].

From 14 high TB, TB-HIV, and mutli-drug resistant TB (MDR-TB) countries worldwide, eight are from Africa including Ethiopia. High HIV burdens [3-5], poor access and quality of healthcare services $[3,6,7]$, poverty $[1,7]$ and high population growth and mobility $[1,3,8]$ are major factors associated with high ongoing TB burdens in developing countries. TB is among the top ten public health problems in Ethiopia with varying magnitudes across regions, zones and districts [9, 10]. TB is a problem of both urban and rural populations in Ethiopia [11, 12]. Poor accessibility of TB diagnostic and treatment services $[9,13,14]$, poverty $[9,14,15]$, high HIV burden $[4,10,16]$, low community awareness [15, 17], and poor TB program monitoring and supports [7, 15] have contributed more to the presence of high TB burdens in Ethiopia.

Early case detection and treatment of the detected cases are the most effective strategies of TB control programs [1]. However, their performance is very far from the expected level globally and worse in high TB burden countries $[1,12,15]$. As a result, more TB cases remain undetected, detected cases did not get full service of anti-TB treatment, and developing countries are suffering from poor treatment outcomes that lead to MDR-TB $[1,3,11,18]$. The global TB control programs have two phases of anti-TB treatment through sputum smear follow-up at the end of the intensive phase and $5^{\text {th }}$-month treatment [19]. This is to check the effectiveness of anti-TB treatment follow-up, take action on-treatment to follow up, and know the treatment outcome status of each TB patient [20]. Although most TB cases are expected to become smear-negative after taking drugs of the intensive phase treatment, a significant number of TB cases remain bacteriologically positive [20]. This increases the length of infectious period of PTB cases [21]. 
Most of the developing countries lacked information on the time to smear conversion due to poor documentation, absence of TB diagnostic tools, and poor TB program monitoring strategies [18, 20, 21]. Similarly, identifying determinant factors that resulted in delayed sputum smear conversion is also vital to improve the effectiveness of TB treatment follow up [20, 22, 23]. The situation has no exception to the Ethiopian TB prevention and control programs [21]. Little is known about the time to sputum smear conversion among smear-positive PTB cases (SPPTB). Although there are few studies, they are among the MDR-TB patients [23]. In addition, we are not aware of factors that lengthen the sputum smear conversion time.

Therefore, we conducted a study on time to sputum smear conversion and associated factors among SPPTB patients in East Gojjam zone, Northwest Ethiopia. The reason for choosing this zone is due to its high TB burden [21, 24], lowest case detection [24], and no such former studies there. Thus, study findings will be important evidence to district, zonal and regional level TB control programs to make informed decisions to control factors and improve the performance of TB control programs. It will also be relevant literature to the coming researchers in TB prevention and control.

\section{Methods and materials}

\section{Study design and settings}

A facility-based prospective longitudinal study was conducted between January-June 2019 among 282 newly diagnosed SPPTB patients in East Gojjam Zone of the Amhara Region, Northwest Ethiopia. The East Gojjam zone, one of the 12 Zones in the Amhara regional state, has a total estimated population of 2,740,625 in 2019. About $85 \%$ of the zonal population is living in rural areas. The zone has about $14,010 \mathrm{~km}^{2}$ area coverage divided into 19 administrative woredas (a third-level administrative hierarchy in Ethiopia). The zone has about 517 public (406 health posts, 102 health centers, and nine hospitals) with a history of offering TB services at the time of data collection [13, 24]. The zone consisting of all types of climate conditions and geographic features.

\section{Source and study population}

All the newly diagnosed SPPTB patients in the intensive phase of anti-TB treatment were the source population. Whereas, all the adult PTB cases (aged $\geq 15$ years) in the intensive phase of anti-TB treatment in the selected health facilities were the study population. The reason for excluding PTB cases aged $<15$ years was related to their physiology and ability to offer purulent sputum for smear follow-up. Usually, children under 15 years have a problem of giving purulent sputum and most are 
extrapulmonary and smear-negative [1, 25]. PTB cases who were seriously ill and unable to understand and respond to questions were also excluded from being study samples.

\section{Sample size determination and sampling techniques}

The sample size (282) was calculated by Epi Info version 7 using a total of 763 smear-positive PTB cases from the study area in 2019 [24], 95\% confidence interval, 5\% margin of error, 50\% PTB proportion since no former published study on sputum smear conversion time among newly diagnosed PTB patients and 10\% non-response rate. To select study health facilities, we considered the rule of thumb and resource constraints to include $25 \%$ of health facilities offering TB services (102 HCs and nine hospitals). According to the Ethiopian healthcare delivery system [26], one health center and five HPs around that HC are termed as primary healthcare unit (PHCU). Based on that, 158 health facilities (two hospitals, $26 \mathrm{HCs}$ and 130 health posts) were included in the study. Accordingly, we included 26 PHCUs and two hospitals. The sample size was allocated to those hospitals and PHCUs proportionally based on the number of smear-positive PTB cases they had during the data collection period.

\section{Data collection tools and procedures}

Data on socio-demographic, clinical profile and personal behavior-related variables of SPPTB patients were collected using an interviewer-administered questionnaire (S1-quesionnaire). The questionnaire was developed by referring to former similar studies on factors of sputum conversion $[20,22,23]$. The questionnaire was pretested among SPPTB patients from health facilities outside of the study area but having the same characteristics. Necessary modifications on order, clarity, relevance and completeness of questions were made based on a pretest result. Six trained clinical nurses and two public health supervisors collected data through a face-to-face interview of each smear-positive PTB patient. Data were collected after getting written informed consent from each study participant.

\section{Sputum sample collection and processing}

A morning sputum specimen was collected from each study participant weekly for 22 weeks duration. Three trained laboratory technologists collected and processed the sputum specimens following the Ethiopian national TB diagnosis, treatment and follow up guideline adapted from the WHO guideline $[8,16]$. Due to the absence of sputum culture and rapid TB diagnostic services in the health facilities of the study area, sputum smears were processed by Ziehl-Neelsen acid-fast bacilli (AFB) staining technique and examined for the presence of bacilli microscopically. A sputum concentration 
technique using a 5\% sodium hypochlorite solution was used to process sputum samples to increase the concentration and detection of bacilli. We used labeled sputum cap, applicator stick, 5\% sodium hypochlorite, distilled water, centrifuged, AFB reagents, plastic bulb pipette, slide, cover slide, immersion oil and Olympus microscope to process sputum specimens. A $2 \mathrm{ml}$ sputum sample was transferred to a test tube of $15-20 \mathrm{ml}$ volume. An equal volume of sodium hypochlorite (bleach) solution was added to a test tube that contains a $2 \mathrm{ml}$ sputum sample and mixed well. Then the mixture was left for 20-30 minutes at room temperature through frequent shaking to break down the mucus in the sputum. Afterward, $8 \mathrm{ml}$ distilled water was added to the mixture, mixed well and centrifuged at 3000rpm for 15minutes. Lastly, the supernatant fluid was removed using a plastic bulb pipette and the smear was prepared from the sediment through the Ziehl Neelsen AFB staining technique [16, 27]. The three trained laboratory technologists examined all prepared smears turn by turn using an Olympus microscope and final results were reported after the approval of three technologists.

\section{Data quality assurance}

Providing two days of training for data collectors, pretesting of a questionnaire, regular supportive supervision of data collectors, double data entry, labeling sputum samples and smear slides with patient's identification number, checking data completeness, and processing sputum samples according to the national and WHO TB diagnosis, treatment and follow up guidelines were some of the data quality control activities. In addition, data collectors and supervisors used a data collection guide book, and the result of each sputum smear was reported after being examined by three laboratory technologists orderly. The sputum-sodium hypochlorite mixture was shaken well and stand on a rack for 20 -30 minutes to breakdown the mucus in the sputum.

\section{Data processing and analysis}

Data were entered, cleaned and analyzed using SPSS version 25. Various types of descriptive statistics such as mean, median, interquartile range, proportions and cross-tabulations were computed to describe study variables. Treatment outcome was measured in terms of favorable (cured) and unfavorable (failed). Each processed sputum smear was examined and reported for each SPPTB patient for 22 weeks duration. Knowledge of each respondent was measured based on the mean score of 12 knowledge questions. Based on that, respondents who scored above the mean score were grouped as having good knowledge about TB. The SPPTB patients who had a history of cigarette smoking of any type, dose and frequency were considered as smokers. Similarly, SPPTB patients who had a history of taking any type of alcohol and amount were considered as alcohol drinkers. 
Moreover, SPPTB patients who had chewed chat with any type, dose, and frequency were grouped as chat chewers. The reasons for considering any type, amount (dose), and duration for personal behaviors were just by assuming they are risky for TB patients on anti-TB treatment in the form of drug-substance reactions, discomforts, forget to take anti-TB drugs and appointments (check-up and collecting anti-TB drugs). The sputum smear conversion time was measured using median time with IQR and proportions. Nutritional status was measured in terms of Body mass index (BMI). PTB patients who had a BMI of $\leq 8 \mathrm{~kg} / \mathrm{m} 2$ were considered as undernutrition and those who had $>18$ $\mathrm{kg} / \mathrm{m} 2$ were grouped as normal since normal and overweight nutritional status have inversely association with TB infection. Factors associated with sputum smear conversion time were identified using bivariate and multivariable logistic regression analysis. The regression associations were described using odds ratio at $95 \%$ confidence interval (CI) and variables were considered as statistically significant if they have a p-value $<0.05$.

\section{Ethical consideration}

The study was conducted according to the principles of the Declaration of Helsinki and fulfilled the Ethiopian National Health Research and Ethics Guideline. The study was conducted after taking ethical clearance and approval from the ethical review committee of the College of Medicine and Health Sciences, Bahir Dar University (Protocol No: 091/18-04). Data were collected after getting written informed consent from each study participant, parents and/or caregivers. Participation was fully voluntary based including not responding after reading the instructions and questions. Data confidentiality was maintained through anonymity by avoiding any personal identifiers. The Amhara Regional Health Bureau and in line offices gave supporting letters after being informed about research objectives and procedures.

\section{Results}

\section{Socio-demographic and personal behaviors of study participants}

A total of 282 newly diagnosed active SPPTB cases, 166(59\%) males and 147(52\%) rural dwellers were followed for up to 22 weeks through sputum smear examination. The mean age of respondents was $35 \pm 5$ SD years. Over a third, 104(37\%) of respondents cannot read and write in education status. About 119(42\%) of SPPTB cases had good knowledge about TB and 42(15\%) of them were HIV positive. Treatment success rate (cured) since we followed up to the end of the $5^{\text {th }}$-month treatment was $95 \%$. Only 54 (19\%) of PTB cases had $\leq 14$ days of cough duration before being diagnosed as SPPTB. A significant number of SPPTB cases (38\%) faced societal stigma and discrimination from 
families, friends and the community. A large number of SPPTB cases, $124(44 \%)$ had greater than or equal to the median TB service delay ( $\geq 82$ days). Over two-thirds, 198(70\%) of the respondents had body mass index values indicating undernutrition (Table1).

Table 1: Socio-demographic, clinical profile and personal behaviors of smear-positive PTB patients in East Gojjam Zone, Northwest Ethiopia, 2019 ( $(\mathbf{n}=\mathbf{2 8 2})$.

\begin{tabular}{|c|c|c|}
\hline Variable & Response & Number (\%) \\
\hline Age in years & $\begin{array}{l}15-35 \\
>35\end{array}$ & $\begin{array}{l}127(45.0) \\
155(55.0)\end{array}$ \\
\hline Sex & $\begin{array}{l}\text { Male } \\
\text { Female }\end{array}$ & $\begin{array}{l}166(59.0) \\
116(41.0)\end{array}$ \\
\hline Residence & $\begin{array}{l}\text { Rural } \\
\text { Urban }\end{array}$ & $\begin{array}{l}147(52.0) \\
135(48.0)\end{array}$ \\
\hline Education level & $\begin{array}{l}\text { Cannot read and write } \\
\text { Can read and write }\end{array}$ & $\begin{array}{l}104(37 \%) \\
178(63.0)\end{array}$ \\
\hline Previous TB history & $\begin{array}{l}\text { Yes } \\
\text { No }\end{array}$ & $\begin{array}{r}57(20.0) \\
225(80.0)\end{array}$ \\
\hline Marital status & $\begin{array}{l}\text { Single } \\
\text { In union }\end{array}$ & $\begin{array}{l}147(52.0) \\
135(48.0)\end{array}$ \\
\hline Knowledge about TB & $\begin{array}{l}\text { Good } \\
\text { Poor }\end{array}$ & $\begin{array}{l}119(42.0) \\
163(58.0)\end{array}$ \\
\hline Grade/bacilli load & $\begin{array}{l}<2+ \\
\geq 2+\end{array}$ & $\begin{array}{l}175(62.0) \\
107(38.0)\end{array}$ \\
\hline HIV status & $\begin{array}{l}\text { Positive } \\
\text { Negative }\end{array}$ & $\begin{array}{r}42(15.0) \\
240(85.0)\end{array}$ \\
\hline Treatment outcome & $\begin{array}{l}\text { Favorable } \\
\text { Unfavorable }\end{array}$ & $\begin{array}{r}268(95.0) \\
14(5.0)\end{array}$ \\
\hline Had diabetes mellitus & $\begin{array}{l}\text { Yes } \\
\text { No }\end{array}$ & $\begin{array}{r}51(18.0) \\
231(82.0)\end{array}$ \\
\hline Had a history of smoking & $\begin{array}{l}\text { Yes } \\
\text { No }\end{array}$ & $\begin{array}{r}54(20.0) \\
214(80.0)\end{array}$ \\
\hline Had a history of chat chewing & $\begin{array}{l}\text { Yes } \\
\text { No }\end{array}$ & $\begin{array}{r}28(10.0) \\
254(90.0)\end{array}$ \\
\hline Had a history of taking alcohol & $\begin{array}{l}\text { Yes } \\
\text { No }\end{array}$ & $\begin{array}{r}73(26.0) \\
209(74.0)\end{array}$ \\
\hline Duration of cough & $\begin{array}{l}\leq 14 \text { days } \\
>14 \text { days }\end{array}$ & $\begin{array}{r}54(19.0) \\
228(81.0)\end{array}$ \\
\hline Faced societal stigma & $\begin{array}{l}\text { Yes } \\
\text { No }\end{array}$ & $\begin{array}{l}107(38.0) \\
175(62.0)\end{array}$ \\
\hline Body mass index (BMI) & $\begin{array}{l}\text { Under nutrition } \\
\text { Normal }\end{array}$ & $\begin{array}{r}198(70.0) \\
84(30.0)\end{array}$ \\
\hline Length of TB service delay & $\begin{array}{l}<82 \text { days } \\
\geq 82 \text { days }\end{array}$ & $\begin{array}{l}158(56.0) \\
124(44.0) \\
\end{array}$ \\
\hline
\end{tabular}

\section{Proportion of sputum conversion time of PTB cases}


The proportions of sputum smear conversion at the end of $1^{\text {st }}, 2^{\text {nd }}, 3^{\text {rd }}, 4^{\text {th }}$, and $5^{\text {th }}$ months of anti-TB treatment follow up periods were 60\% (95\%CI: 52\% -72\%), 85\% (95\%CI: 76\% -92\%), 92\% (95\%CI: 80 -96), 94\% (95\% CI: 85 -98), and 95\% (95\% CI: 85\% -99\%), respectively (Figure 1).

Figure 1: Proportion of sputum smear conversion at the end of $1^{\text {st }}, 2^{\text {nd, }} 3^{\text {rd }}, 4^{\text {th }}$, and $5^{\text {th }}$ months anti-TB treatment follow up in East Gojjam Zone, Northwest Ethiopia, 2019.

Based on the anti-TB treatment follow up of each PTB cases for 22 consecutive weeks, the median sputum smear conversion time was found to be 35 days, IQR: 21 -56 days (Figure2).

Figure2: Trend of time to sputum smear conversion over 22 weeks follow up period, 2019.

\section{Factors associated with sputum smear conversion time}

The multivariable logistic regression analysis indicated that BMI, smear grading, HIV infection, having DM, knowledge on TB, cigarette smoking, faced societal stigma, and length of TB service delay were statistically significant factors $(\mathrm{P}$-value < 0.05$)$ of smear conversion time in the study area. Accordingly, the odds of having higher sputum conversion time was double among PTB cases with undernutrition status compared to PTB cases with normal and overweight people $(\mathrm{AOR}=2.01$, $95 \% \mathrm{CI}=1.31-3.85)$. PTB cases having initial smear grading of $<2+$ were $54 \%$ times less likely to have delayed sputum smear conversion time compared to PTB cases with $\geq 2+$ sputum smear grading $(\mathrm{AOR}=0.46,95 \% \mathrm{CI}=0.21-0.73)$. In addition, PTB cases who had HIV and DM positive status were twice to have delayed sputum smear conversion compared to the counterpart PTB cases (AOR $=2.38,95 \% \mathrm{CI}=1.20-4.91$, and $\mathrm{AOR}=2.11,95 \% \mathrm{CI}=1.18-4.20)$, respectively.

Similarly, PTB cases with cigarette smoking history and who faced societal stigma due to their TB status were double times to have delayed sputum smear conversion time compared to their counterpart PTB cases $(\mathrm{AOR}=1.96,955 \mathrm{CI}=1.20-3.82$, and $\mathrm{AOR}=2.0,95 \% \mathrm{CI}=1.26-3.52)$, respectively. Moreover, the odds of having delayed sputum smear conversion time was $65 \%$ times less likely among PTB cases with lower TB service delay compared to PTB cases who had longer TB service delay; $\mathrm{AOR}=0.35,95 \% \mathrm{CI}=0.23-0.64$ (Table 2$)$. 
Table2: Factors associated with sputum smear conversion in East Gojjam zone, Ethiopia, 2019 (n = 268).

\begin{tabular}{|c|c|c|c|c|c|}
\hline \multirow{2}{*}{ Variable } & \multirow{2}{*}{ Response } & \multicolumn{2}{|c|}{ Smear conversion time } & \multirow{2}{*}{ COR $(95 \% \mathrm{CI})$} & \multirow{2}{*}{$\operatorname{AOR}(95 \% \mathrm{CI})$} \\
\hline & & $>35$ days & $\leq 35$ days & & \\
\hline \multirow[t]{2}{*}{ Age in years } & $15-35$ & $60(22.4)$ & $67(25.0)$ & $0.96(0.59-1.55)$ & $0.68(0.35-1.42)$ \\
\hline & $>35$ & $68(25.4)$ & $73(27.2)$ & 1 & 1 \\
\hline \multirow[t]{2}{*}{ Sex } & Male & $75(28.0)$ & $83(31.0)$ & $0.97(0.60-1.58)$ & $1.35(0.26-2.15)$ \\
\hline & Female & $53(19.7)$ & $57(21.3)$ & 1 & 1 \\
\hline \multirow[t]{2}{*}{ Residence } & Rural & $62(23.0)$ & $78(29.0)$ & $0.75(0.46-1.23)$ & $0.41(0.15-1.08)$ \\
\hline & Urban & $66(25.0)$ & $62(23.0)$ & 1 & 1 \\
\hline \multirow[t]{2}{*}{ Education level } & Cannot read \& write & $45(17.0)$ & $54(20.0)$ & $0.86(0.53-1.42)$ & $1.58(0.32-2.71)$ \\
\hline & Can read and write & $83(31.0)$ & $86(32.0)$ & 1 & 1 \\
\hline \multirow[t]{2}{*}{ Marital status } & Single & $63(23.5)$ & $77(28.7)$ & $0.79(0.49-1.28)$ & $0.48(0.23-1.12)$ \\
\hline & In union & $65(24.3)$ & $63(23.5)$ & 1 & 1 \\
\hline \multirow[t]{2}{*}{ Prior TB history } & Yes & $24(9.0)$ & $30(11.2)$ & $0.85(0.46-1.54)$ & $0.52(0.22-1.29)$ \\
\hline & No & $110(41.0)$ & $104(38.8)$ & 1 & 1 \\
\hline \multirow[t]{2}{*}{ Body mass index } & $\leq 18 \mathrm{~kg} / \mathrm{m} 2$ & $101(37.7)$ & $87(32.5)$ & $2.30(1.32-3.93)$ & $2.01(1.31-3.85)$ \\
\hline & $>18 \mathrm{~kg} / \mathrm{m} 2$ & $27(10.1)$ & $53(19.7)$ & 1 & 1 \\
\hline \multirow[t]{2}{*}{ Duration of cough } & $\leq 14$ days & $28(10.4)$ & $23(8.6)$ & $1.42(0.77-2.63)$ & $0.86(0.58-2.15)$ \\
\hline & $>14$ days & $100(37.3)$ & 117 (43.7) & 1 & 1 \\
\hline \multirow[t]{2}{*}{ Smear grading } & $<2+$ & $68(25.4)$ & $98(36.6)$ & $0.49(0.29-0.81)$ & $0.46(0.21-0.73)$ \\
\hline & $\geq 2+$ & $60(22.4)$ & $42(15.7)$ & 1 & 1 \\
\hline \multirow[t]{2}{*}{ HIV status } & Positive & $27(10.1)$ & $13(4.8)$ & $2.61(1.28-5.32)$ & $2.38(1.20-4.91)$ \\
\hline & Negative & $101(37.7)$ & $127(47.4)$ & 1 & 1 \\
\hline \multirow[t]{2}{*}{ Having DM } & Yes & $31(11.5)$ & $17(6.3)$ & $2.31(1.21-4.42)$ & $2.11(1.18-4.20)$ \\
\hline & No & $97(36.2)$ & $123(46.0)$ & 1 & 1 \\
\hline \multirow[t]{2}{*}{ Knowledge on TB } & Good & $42(15.7)$ & $71(26.5)$ & $0.47(0.29-0.78)$ & $0.44(0.23-0.69)$ \\
\hline & Poor & $86(32.0)$ & $69(25.8)$ & 1 & 1 \\
\hline \multirow[t]{2}{*}{ Cigarette smoking } & Yes & $34(12.7)$ & $20(7.5)$ & $2.20(1.25-4.01)$ & $1.96(1.20-3.82)$ \\
\hline & No & $94(35.0)$ & $120(44.8)$ & 1 & 1 \\
\hline \multirow[t]{2}{*}{ Taking alcohol } & Yes & $34(12.7)$ & $36(13.4)$ & $1.05(0.61-1.81)$ & $0.82(0.48-1.58)$ \\
\hline & No & $94(35.1)$ & $104(38.8)$ & 1 & 1 \\
\hline \multirow[t]{2}{*}{ Faced stigma } & Yes & $61(22.7)$ & $41(15.3)$ & $2.20(1.33-3.64)$ & $2.0(1.26-3.52)$ \\
\hline & No & $67(25.0)$ & $99(40.0)$ & 1 & 1 \\
\hline Length of TB & $<82$ days & $54(20.0)$ & $96(36.0)$ & $0.33(0.21-0.55)$ & $0.35(0.23-0.64)$ \\
\hline service delay & $\geq 82$ days & $74(27.6)$ & $44(16.4)$ & 1 & 1 \\
\hline
\end{tabular}




\section{Discussion}

Sputum smear negativity (become non-infectious) after a certain period of anti-TB treatment initiation is the best marker to evaluate the response of anti-TB treatment. Various literature showed different lengths of sputum and culture conversion time [20,22]. This study revealed a median sputum smear conversion time of 35 days, IQR: 33 -68 days. This was in line with former studies that reported a median time of 35 days [28] and 34 days with an IQR of 28 -54 [23]. Alternatively, this finding was lower compared to a study finding from Afghanistan [20] where the sputum smear conversion time was 60 days. The observed discrepancy might be related to variations in the study period, socioeconomic status of patients, and strength of TB control programs. The current finding was also found to be higher from findings of previous studies elsewhere; median time of 24 days with IQR $12-54$ [22] and 21 days ranging from 5 to 141 days [29]. The discrepancy might be related to the difference in length of the follow-up period to estimate the infectious period, characteristics of PTB cases (personal behaviors, nutritional status and adherence to anti-TB drugs), and clinical profile of PTB cases including comorbidity status (HIV and DM). All these can determine the size of period of noninfectious after treatment initiation.

In most studies, the proportion of sputum smear conversion at the end of the $5^{\text {th }}$ treatment was consistent with our findings $[22,23,28]$. The proportions of smear conversion at the end of the $2^{\text {nd }}$ month of treatment on the other hand showed discrepancies with the former studies. Accordingly, this finding $(85 \%)$ was found to be higher compared to proportions of smear conversions from Ethiopia [23], Italy [22], and Iran [30] where proportions of smear conversion were (81.6\%), (78.7\%) and $83.6 \%$ at the end of $2^{\text {nd }}$-month treatment, respectively. This might be related to the use of concentration techniques to process sputum samples in our study that have better sensitivity to increase positivity than the routine sputum smear processing [27]. In contrast, it was lower from study findings elsewhere where proportions of sputum smear conversions were 93.4\% [31] and 95\% [32] by the end of the $2^{\text {nd }}$-month treatment. Differences in the socio-economic status of TB patients, quality of TB monitoring strategies, comorbidity status, personal behaviors, and complications of illness might result in these discrepancies. In our study, the trend of sputum smear conversions showed a high increase between the end of the $1^{\text {st }}$ month and $2^{\text {nd }}$ months and slight increasing to the end of $5^{\text {th }}$ month of treatment follow up (Figure1). This is possibly linked to the effectiveness of bacteriocidic anti-TB drugs given in the intensive phase that aimed to lower bacterial load [19]. 
Based on the multivariable logistic regression analysis, PTB cases who had $\leq 2+$ sputum smear grade were $54 \%$ times less likely to have delayed sputum smear conversion compared to PTB cases with > $2+$ smear grading. The possible explanation is linked to the high load of bacilli with PTB cases having higher smear grades that require time to be cleared and become zero compared to the lower one making other conditions constant. Prior studies from elsewhere reported similar results [20, 22, 28].

The odds of having longer sputum smear conversion was twice among PTB cases who had HIV and DM infections compared to their counterpart PTB cases. This was supported by previous studies from different parts of the world [20, 29, 30,33]. This is attributed by diseases complications, poor antiTB treatment adherence, drug interactions, declined immunity status, undernutrition due to poor dietary intake, psychological stress, and societal stigma. All these can determine treatment adherence and sputum conversion needs immune-competent PTB patients [3]. This implies that timely integrated screening and management of comorbidities such as TB, HIV and DM is required to improve the performance of TB prevention and control programs. This might prevent TB service delays, poor treatment outcomes, length of TB infectious period, and transmission to others.

Similarly, PTB patients with undernutrition were double times to have delayed sputum smear conversion compared to normal and overweight PTB patients. Former studies also reported undernutrition as a predictor variable to longer sputum smear conversion time [23, 29, 34, 35]. The possible explanation for this is due to the contribution of undernutrition to have weakened immunity status that makes people more susceptible to get TB infection, have high disease complications, and less chance of sputum smear conversion [3].

In this study, knowledge of PTB patients on TB was found to be a statistically significant factor in sputum smear conversion time. Based on that, PTB patients having good knowledge were $56 \%$ times less likely to have delayed sputum smear conversion compared to PTB patients with good knowledge of TB. This might be linked to better anti-TB treatment adherence that people with better knowledge will do. If TB patients are aware of TB (prevention, diagnosis and treatment), they will relatively have better TB service seeking practice and adherence in anti-TB treatment follow up [17]. This will then result in having non-delayed sputum smear conversion time.

In addition, the smoking habit of PTB patients was reported as statistically significant with the length of sputum smear conversion time. Thus, the odds of having long sputum smear conversion time was twice among PTB patients who smoked a cigarette in any amount and frequency compared to non- 
smoker PTB patients. Previous studies on TB and smoking also reported supporting result [35-37]. This is attributed to the effect of smoking on lung defense mechanisms, lowering T-cell immunities, and poor dietary intake. These make people immune incompetent that makes them more susceptible to TB infection, high TB disease complications, and poor treatment adherence due to complications, drug -substance interactions, forgetfulness to take and collect anti-TB drugs [21, 37]. All these lead to have delayed sputum smear conversion and poor treatment outcomes including death.

Moreover, PTB cases that faced societal stigma had double odd of getting delayed sputum smear conversion time than the counterpart PTB cases. Most people with less awareness about TB think that TB and HIV are inseparable. As a result, they have poor TB service-seeking practices and make stigmatization towards TB patients by assuming they have also HIV infection. This makes TB patients to be isolated from social events and friends. This results in getting poor supports, poor treatment adherence, psychological stress and TB disease complications [15, 21]. Such conditions most likely end with poor sputum smear conversion time.

The length of TB service delay (patient and facility) also showed a positive association with the length of non-deferred stuprum smear conversion time. Consequently, PTB patients with lower TB service delay were $65 \%$ times less likely to have deferred sputum conversion time than PTB patients with delayed TB service seeking practices. Longer delay in seeking TB services results in disease complications, longer infectious period and weakened immunity that might lead to other infections including HIV, DM and undernutrition [7, 21, 38]. Thus, people who started anti-TB treatment after a longer TB service delay might have poor treatment response including deferred sputum conversion.

\section{Strength and limitations of the study}

The strength of this study relies on employing the sputum concentration technique to increase the sensitivity of sputum smear detection. Estimating the proportions of sputum smear conversion at more points of treatment follow-up period might also be considered as a strength since it has a significant role in the monitoring of treatment follow up periods. Due to health education about TB and its treatment, and strong counseling and follow up of TB cases, we make defaulting from treatment zero with a 95\% cure rate. The study also identified important variables that need to be considered to improve treatment outcomes among TB patients. 
However, using sputum smear conversion due to the absence of sputum culture and other rapid TB diagnostic services might have little impact on estimating the size of proportions and median time of sputum smear conversion time. Thus, interpretation of findings needs to account for this limitation.

\section{Conclusions}

Although the treatment success rate was better (95\%), the median sputum smear conversion time was higher compared to the TB program expectations and some former studies. Undernutrition, high prior smear grade, being HIV positive, having DM, poor knowledge on TB, cigarette smoking, facing stigma and length of TB service delay were statistically significant factors to the length of sputum smear conversion time. The highest effort is needed to improve the health literacy of the community by revising the existing community awareness strategies is essential to improve treatment adherence and lower sputum smear conversion time. This will improve their knowledge on TB, and shape their nutritional status, personal behaviors and societal stigma. Early management of comorbidities through offering integrated services for TB, HIV and DM is required. A further study that considers large sample size and sputum culture is suggested to estimate the size sputum smear conversion time.

\section{Abbreviation}

AFB: Ziehl-Neelsen Acid-Fast Bacilli, AOR: Adjusted odds ratio, BMI: body mass index, CI: confidence interval, COR: crude odds ratio, DM: diabetes mellitus, HCs: health centers, IQR: interquartile range, MDR-TB: multi-drug resistant tuberculosis, PHCU: primary health care unit, PTB: pulmonary tuberculosis, SD: standard deviation, and SPPTB: smear-positive PTB.

\section{Declarations}

\section{Ethics approval and consent to participate}

Ethical clearance was taken from the ethical review committee of the College of Medicine and Health Sciences, Bahir Dar University (Protocol No: 091/18-04). Data were collected after getting written informed consent from each study participant, and their parents and/or legal caregivers. Participation was fully voluntary based including not responding after reading the instructions and questions. Data confidentiality was maintained through anonymity by avoiding any personal identifiers.

\section{Consent for publication}

Not applicable 


\section{Availability of data and materials}

The datasets used and/or analyzed during the current study are available from the corresponding author on reasonable request.

\section{Competing interests}

The author declares as there are no competing interests in this work.

\section{Funding}

Bahir Dar University covered the budget for data collection (grant No: RCS/254/09), but it has no technical role in the research processes.

\section{Author's contributions}

The author performed each activity of the manuscript from commencement to final approval with the consultation of senior researchers in the field. Lastly, the author read and approved the manuscript.

\section{Acknowledgments}

The researcher would like to forward the deepest appreciation to Bahir Dar University for covering the data collection budget. The author also gave gratitude to TB program officers at different levels, PTB patients, data collectors, and supervisors for their meaningful supports during data collection.

\section{Author's information}

${ }^{1} \mathrm{PhD}$ in public health, associate professor of Public Health in the School of Public Health, College of Medicine and Health Sciences, Bahir Dar University, Bahir Dar, Ethiopia.

Email: muler.hi@gmail.com or Mulusew.Andualem@bdu.edu.et 


\section{References}

1. World Health Organization: Global Tuberculosis report 2020. Geneva:Licence: CC BY-NC-SA 3.0 IGO. 2020.

2. Glaziou P, Sismanidis C, Floyd K, Raviglione M. Global epidemiology of tuberculosis. Cold Spring Harbor perspectives in medicine. 2015; 5(2):a017798.

3. Turner RD, Chiu C, Churchyard GJ, Esmail H, Lewinsohn DM, Gandhi NR, Fennelly KP. Tuberculosis Infectiousness and Host Susceptibility. The Journal of Infectious Diseases. 2017; 216(S6): S636-643.

4. Gelaw YA, Williams G, Soares Magalhaes RJ, Gilks CF, Assefa Y. HIV Prevalence Among Tuberculosis Patients in Sub-Saharan Africa: A Systematic Review and Meta-analysis. AIDS and behavior. 2019; 23(6):1561-1575.

5. World Health Organization. TB and HIV and other comorbidities. https://www.who.int/tb/areas-ofwork/tb-hiv/en/\#: :text=TB\%20 and\%20 HIV\%2C\%20and\%20the,cause\%20of\%20HIV\%2Drelated\%20 deaths. 2018.

6. De Schacht C, Mutaquiha C, Faria F, Castro G, Manaca N, Manhica I, Cowan J. Barriers to access and adherence to tuberculosis services, as perceived by patients: A qualitative study in Mozambique. PloS one. PloS one. 2019; 14(7).

7. Getnet $F$, Demissie $M$, Assefa N, Mengistie B, Worku A. Delay in the diagnosis of pulmonary tuberculosis in low-and middle-income settings: systematic review and meta-analysis. $B M C$ Pulmonary Medicine. 2017; 17(202).

8. Ethiopian Federal Ministry of Health. Comprehensive training manual for clinical and programmatic management of TB/L and TB/HIV Participants' manual, Addis Ababa. 2017.

9. Gelaw YA, Williams G, Assefa Y, Asressie M, Soares Magalhaes RJ. Sociodemographic profiling of tuberculosis hotspots in Ethiopia, 2014-2017. Transactions of the Royal Society of Tropical Medicine and Hygiene. 2019; 113(7):379-391.

10. Kebede AH, Alebachew Z, Tsegaye F, Lemma E, Abebe A, Agonafir M, et.al. The first population-based national tuberculosis prevalence survey in Ethiopia, 2010-2011. The International Journal of Tuberculosis and Lung Disease. 2014; 18(6):635-639.

11. Datiko DG, Guracha EA, Michael E, Asnake G, Demisse M, Theobald S, et. al. Sub-national prevalence survey of tuberculosis in rural communities of Ethiopia. BMC public health. 2019; 19(1):295.

12. Merid Y, Mulate YW, Hailu M, Hailu T, Habtamu G, Abebe M, et.al. Population-based screening for pulmonary tuberculosis utilizing community health workers in Ethiopia. International Journal of Infectious Diseases. 2019; 89:122-127.

13. Asemahagn MA, Alene GD, Yimer SA. Geographic Accessibility, Readiness, and Barriers of Health Facilities to Offer Tuberculosis Services in East Gojjam Zone, Ethiopia: A Convergent Parallel Design. Research and Reports in Tropical Medicine. 2020; 11:3-16.

14. Ereso BM, Yimer SA, Gradmann C, Sagbakken M. Barriers for tuberculosis case finding in Southwest Ethiopia: A qualitative study. PLoS One. 2020; 15(1).

15. Asemahagn MA, Alene GD, Yimer SA. A Qualitative Insight into Barriers to Tuberculosis Case Detection in East Gojjam Zone, Ethiopia. Am J Trop Med Hyg. 2020; 103(4):1455-1465:

doi:1410.4269/ajtmh.1420-0050. 
16. Ethiopian Federal Ministry of Health. Guidelines for clinical and programmatic management of TB, TB/HIV and Leprosy in Ethiopia, sith edition, Addis Ababa. March 2016.

17. Datiko DG, Habte D, Jerene D, Suarez P. Knowledge, attitudes, and practices related to TB among the general population of Ethiopia: Findings from a national cross-sectional survey. PLOS ONE. 2019; 14(10,e0224196.).

18. Cazabon D, Alsdurf H, Satyanarayana S, Nathavitharana R, Subbaraman R, Daftary A, Pai M. Quality of tuberculosis care in high burden countries: the urgent need to address gaps in the care cascade. International Journal of Infectious Diseases. 2017; 56(111 -116:www.elsevier.com/locate/ijid).

19. World Health Organization. Treatment of tuberculosis: guidelines. In. Geneva: World Health Organization. 2010.

20. Azarkar Z, Sharifzadeh G, Ebrahimzadeh A, Olumi S. Time to Sputum Smear Conversion in SmearPositive Pulmonary Tuberculosis Patients and Factors for Delayed Conversion. IJMS. 2016; 14 (1).

21. Asemahagn MA, Alene GD, Yimer SA. Tuberculosis infectious pool and associated factors in East Gojjam Zone, Northwest Ethiopia. BMC Pulmonary Medicine. 2019; 19(229).

22. Bisognin F, Amodio F, Lombardi G, Reggiani MLB, Vanino E, Attard L, et.al. Predictors of time to sputum smear conversion in patients with pulmonary tuberculosis under treatment. New Microbiologica. 2019; 42(3):171 -175,ISN 1121-7138.

23. Tekalegn Y, Woldeyohannes D, Assefa T, Aman R, Sahiledengle B. Predictors of Time to Sputum Culture Conversion Among Drug-Resistant Tuberculosis Patients in Oromia Region Hospitals, Ethiopia This article was published in the following Dove Press journal: Infection and Drug Resistance. Infection and Drug Resistance. 2020; 13: 2547-2556.

24. Amhara National Regional State Health Bureau. The 2018/19 annual performance report of Amhara National Regional State Health Bureau. Bahir Dar. August 2019.

25. Seddon JA, Shingadia D. Epidemiology and disease burden of tuberculosis in children: A global perspective. Infection and drug resistance. 2014; 7:153-165.

26. Ethiopian Federal Ministry of Health. Health sector transformation plan (HSTP): 2015/16-2019/20. Ministry of Health, Addis Ababa, Ethiopia. 2015.

27. Makaen J, Maure T. Bleach Processed Smear for Acid Fast Bacilli Staining in Papua New Guinea. 2014;45. Lab Med Fall. 2014; 45:(4, DOI: 10.1309/LMN45YOZMNPKLRMS).

28. Parikh R, Nataraj G, Kanade S, Khatri V, Mehta P. Time to Sputum Conversion in Smear Positive Pulmonary TB Patients on Category I DOTS and Factors Delaying it. JAPI. 2012; 60.

29. MASUT O, BOISSET S, EPAULARD O. Determinants of time to sputum smear conversion (TSSC) during lung tuberculosis therapy in a French cohort.

30. Behnaz F, Mohammadzadeh M, Mohammadzade G. Five-Year Assessment of Time of Sputum Smears Conversion and Outcome and Risk Factors of Tuberculosis Patients in Central Iran. Tuberculosis Research and Treatment. 2015; Article ID 609083, 7 pages. http://dx.doi.org/10.1155/2015/609083.

31. Djouma FN, Noubom M, Ateudjieu J, Donfack H. Delay in sputum smear conversion and outcomes of smear-positive tuberculosis patients: a retrospective cohort study in Bafoussam, Cameroon. Djouma et al BMC Infectious Diseases. 2015; 15(139,DOI 10.1186/s12879-015-0876-1).

32. Bouti K, Aharmim M, Mouna KM. Factors Influencing Sputum Conversion among Smear-Positive Pulmonary Tuberculosis Patients in Morocco. ISRN Pulmonology. 2013, Volume 2013, Article ID 486507, 5 pages, http://dx.doi.org/10.1155/2013/486507. 
33. Mohd Shariff NM, Safian N. Diabetes mellitus and its influence on sputum smear positivity at the 2 nd month of treatment among pulmonary tuberculosis patients in Kuala Lumpur, Malaysia: A casecontrol study. International Journal of Microbiology. 2015; 4:323 -329, www.elsevier.com/locate/IJMYCO.

34. Assemiea MA, Alenea M, Petruckab P, Leshargied CT, Ketema DB. Time to sputum culture conversion and its associated factors among multidrug-resistant tuberculosis patients in Eastern Africa: A systematic review and meta-analysis. International Journal of Infectious Diseases. 2020; 98:230 -236.

35. Nandasena S, Senavirathna C, Munasinghe C, Wijesena C, Sucharitharathna R. Characteristics and sputum conversion of tuberculosis (TB) patients in Kalutara, Sri Lanka. Indian Journal of tuberculosis. 2019; 66:76 -80. http://www.journals.elsevier.com/indian-journal-of-tuberculosis/.

36. Liu Q, Lu P, Martinez L, Yang H, Lu W, Ding X, Zhu L. Factors affecting time to sputum culture conversion and treatment outcome of patients with multidrug-resistant tuberculosis in China. Liu et al BMC Infectious Diseases. 2018; 18(114.https://doi.org/10.1186/s12879-018-3021-0).

37. Metanat M, Sharifi-Mood B, Parsi M, Sanei-Moghaddam S. Effect of cigarette smoking on sputum smear conversion time among adult new pulmonary tuberculosis patients: A study from Iran Southeast. Iranian Journal of Clinical Infectious Diseases. 2010; 5(1):14 -17.

38. Asres M, Gedefaw M, Kahsay A, Weldu Y. Patients' Delay in Seeking Health Care for Tuberculosis Diagnosis in East Gojjam Zone, Northwest Ethiopia. Am J Trop Med Hyg. 2017; 96(5):1071 -1075 : doi:1010.4269/ajtmh.1016-0892. 
Figures

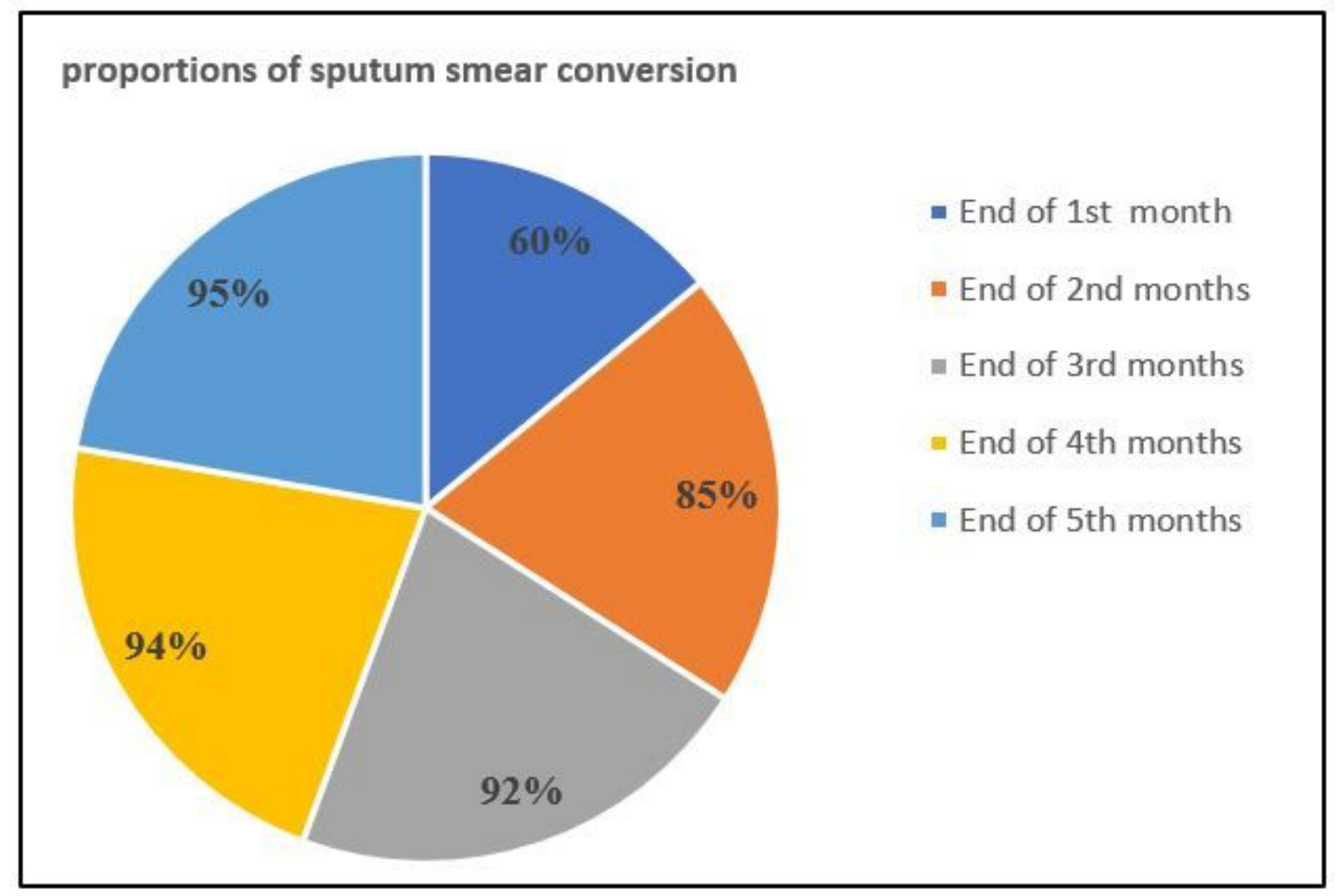

Figure 1

Proportion of sputum smear conversion at the end of $1 \mathrm{st}, 2 \mathrm{nd}$, 3rd, 4th, and 5th months anti-TB treatment follow up in East Gojjam Zone, Northwest Ethiopia, 2019. 


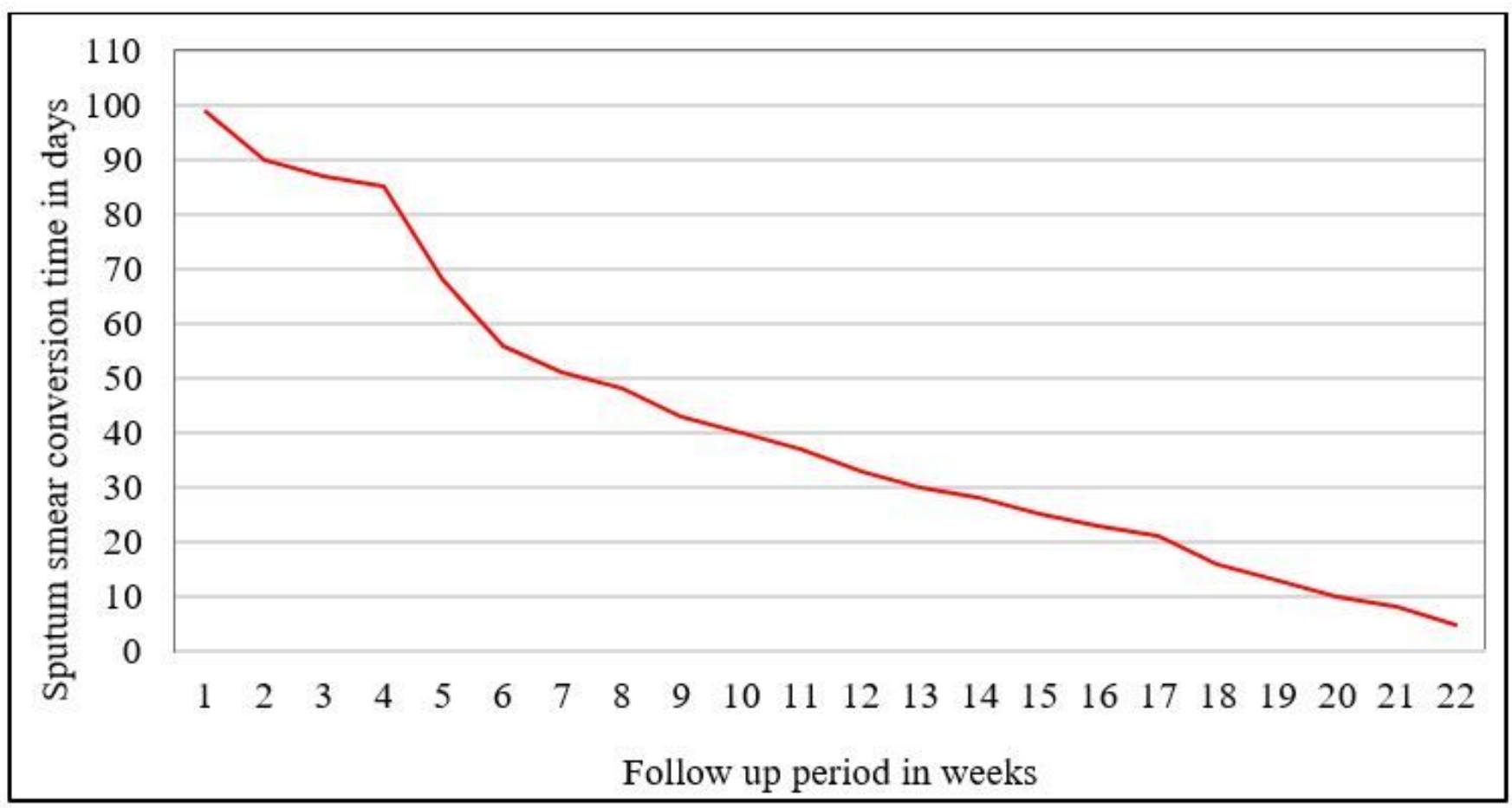

Figure 2

Trend of time to sputum smear conversion over 22 weeks follow up period, 2019.

\section{Supplementary Files}

This is a list of supplementary files associated with this preprint. Click to download.

- S1QustionnaireforTBinfectiousnessaftertreatmentinitiation.docx

- Titlepageforsupplementaryfiles.docx

- S2IQRofPTBconversion.xIsx 